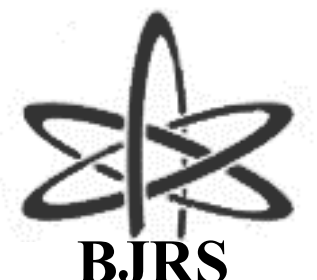

BRAZILIAN JOURNAL

$\mathrm{OF}$

RADIATION SCIENCES

10-01 (2022) 01-16

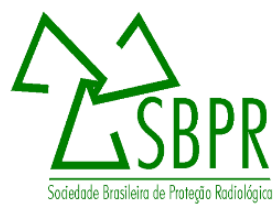

\title{
Deriving formula to simulate the radiological behavior during LOCA in open pool type reactor
}

\author{
Abdelhady A. \\ Reactors Dept., Nuclear Research Center, Atomic Energy Authority, Egypt. \\ amr.abdelhady@gmail.com
}

\begin{abstract}
This study aims to model the radiological consequences that result from loss of coolant accident in open pool research reactor of $22 \mathrm{MW}$ power. The loss of coolant accident results from rupture in the cooling systems of the reactor and consequently results in decreasing the water level in the reactor pool. Decreasing the water level, that represents the shielding material above the reactor core, results in increasing the dose rate in the top region in the reactor pool as well as the reactor building. LOCA normally results in shutting down the reactor due to the radioprotection system provided in the upper region of the reactor pool. A failure in shutdown systems of the reactor during LOCA is rare but expected and it represents the worst case. So, evaluating LOCA from the radiological point of view must be investigated for the safe shutdown mode as well as the failure of shutdown case. The study also includes occurring LOCA for shutdown reactor taking the decay time into consideration.

ORIGEN-S module from scale system was used to estimate the delayed neutrons and gamma rays from the core for the shutdown mode. KENO-VI module from Scale system was used to estimate the prompt neutrons and gamma rays generated from the core for the failure case. Then, MAVRIC/Scale module was used to estimate the dose rate at the top point of the reactor pool for the two cases during LOCA.

Studying the dose rate during LOCA aims to determine the water level that verifies the radiological safety limit at the top point of the reactor pool for the two cases. The calculations introduced a relationship between the water level that verifies the permissible radiological limit $(W L)$ and the decay time $(D T)$ in the range between 0 to
\end{abstract} $30 \mathrm{~d}$.

Keywords: LOCA, Open pool reactor, KENO-VI code, ORIGEN-S code, MAVRIC code, radiation dose rate. 


\section{INTRODUCTION}

Radiation dose rate at the top point of the open pool type reactor is an important point for evaluating the radiological reactor state because it was provided with radiological alarm system that is capable to trigger the reactor shutdown system in case of rising the dose rate. The top point also is located at the bottom of the reactor hall, as shown in figure 1, where the operator is usually found during the most reactor tasks. So, this point corresponds to the maximum dose rate that the operator would be exposed during the accident.

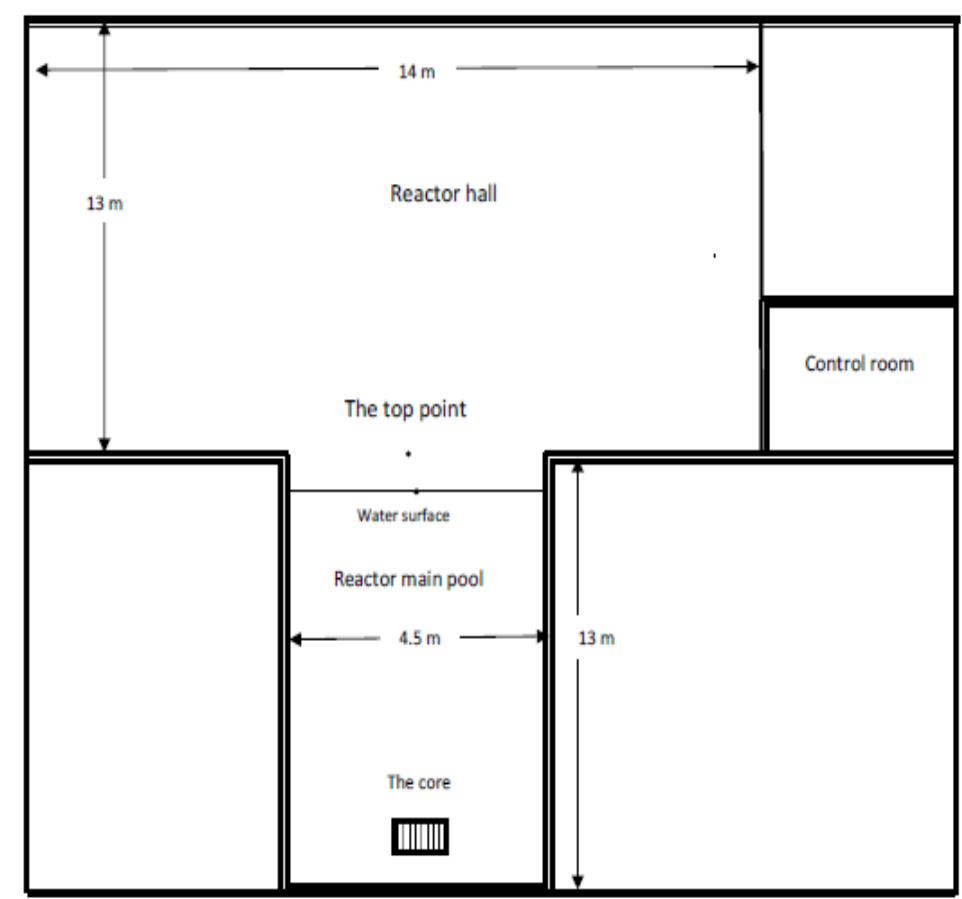

Figure 1: Schematic diagram for reactor building

The radiation dose rate at this point is resulting from two main sources; the radioactive isotopes dissolved in the pool water as well as the neutrons and gamma rays from the reactor core. During normal reactor operation, the dose rate at this point results from the dissolved radioactive isotopes only and the contribution of the core source is negligible due to the high water level above the reactor core which is sufficient to attenuate the neutrons and gamma rays. A hot water layer system was provided to the reactor pool to prevent the dissolved radioactive isotopes to reach the top region 
of the pool and thus reducing the dose rate above the pool water surface [1]. So, the effect of the neutrons and gamma rays from the core in the dose rate at this point is obliged to be accompanied with dropping of water level in the main pool. Dropping of water level may be occurred due to loss of coolant accident (LOCA) owing to rupture in the cooling systems or the neutron beam tubes.

Since evaluating the dose rate resulting from the dissolved radioactive isotopes in the pool water during normal reactor operation has been studied before [1], this study will focus in determining the dose rate resulting from the reactor core in case of LOCA.

LOCA is one of the anticipated accidents that results in increasing the radiation dose rate at the top point of the pool and consequently shutting down the reactor as mentioned above. In spite of providing the reactor with safety systems to shut down the reactor core safely during LOCA, a failure of shutdown systems, that represents the catastrophic case, must be taken into consideration. So, evaluating the dose rate during LOCA accompanied with reactor shutdown as well as failure of reactor shutdown is the objective to determine the water level that achieves the radiological safety for each state.

Evaluating the reactor core source is the main parameter to estimate the dose rate in the top region of the pool. The reactor core, during normal operation mode, is a source of prompt neutrons and gamma rays of intensities depending on the reactor power. During reactor shutdown mode, the source is the delayed neutrons and gamma rays resulting from the fission products.

For simplification, the LOCA accompanied with and without shutdown is abbreviated in the manuscript with (LOCWS) and (LOCNS) respectively.

ORIGEN-S code [2] was used to simulate the delayed neutrons and gamma rays in the LOCWS case. KENO-VI/Scale system [2] was used to simulate the reactor core as a source of prompt neutrons and gamma rays in the LOCNS case. Then, the source was used in MAVRIC/Scale module [2] to determine the dose rate in the top point of the main pool during LOCA.

\section{MATERIALS AND METHODS}

This section discusses the description of the reactor core, the pool tank, and the Scale model that used to simulate the reactor core and the main pool at different water levels. 


\subsection{The main pool and the reactor core description}

The reactor is an open pool research reactor of $22 \mathrm{MW}$ power designed as a multi-purposes reactor to facilitate using the fission neutrons in different applications (material testing, radioisotopes production, neutron physics, and radiography). The reactor contains main and auxiliary open pools. The auxiliary pool is used as spent fuel storage with a maximum capacity of 800 spent fuel elements [3]. The main pool is hollow cylindrical shape tank made from stainless steel with dimension of $13 \mathrm{~m}$ height and internal diameter of $4.5 \mathrm{~m}$. It is filled with de-mineralized water and the core is located near the bottom region of the pool tank covered with water of $11 \mathrm{~m}$ height.

The pool tank is surrounded with a heavy concrete to attenuate the neutrons in the radial direction. The reactor core is surrounded with irradiation boxes, beryllium blocks, and provided with radial and tangential neutron beam tubes as shown in figure 2 . The core is $5 \times 6$ grid that contains 29 fuel elements and cobalt device. The fuel element has 19 fuel plates and each fuel plate contains $\mathrm{U}_{3} \mathrm{O}_{8}$ in a matrix of dispersed aluminum that cladded with AL6061 plates. The coolant channel width is $2.7 \mathrm{~mm}$. Each fuel element contains $404.7 \mathrm{~g}$ of ${ }^{235} \mathrm{U}$ with enrichment of $20 \%$. The fuel plate has a total length of $110 \mathrm{~cm}$ with active length (that contain the $\mathrm{U}_{3} \mathrm{O}_{8}$ fuel) of $80 \mathrm{~cm}$. The plate active zone is $80 \mathrm{~cm} \times 6.4 \mathrm{~cm}$ with a meat thickness of $0.7 \mathrm{~mm}$ and the aluminum cladding thickness of $0.4 \mathrm{~mm}[3]$.

The core is provided with 6 control plates made from a mixture of highly absorbed neutrons (Ag-In-Cd). In case of loss of first shutdown system, a gadolinium injection system would be triggered to fill 4 chambers surrounding the core with gadolinium to shutdown the reactor safely. 


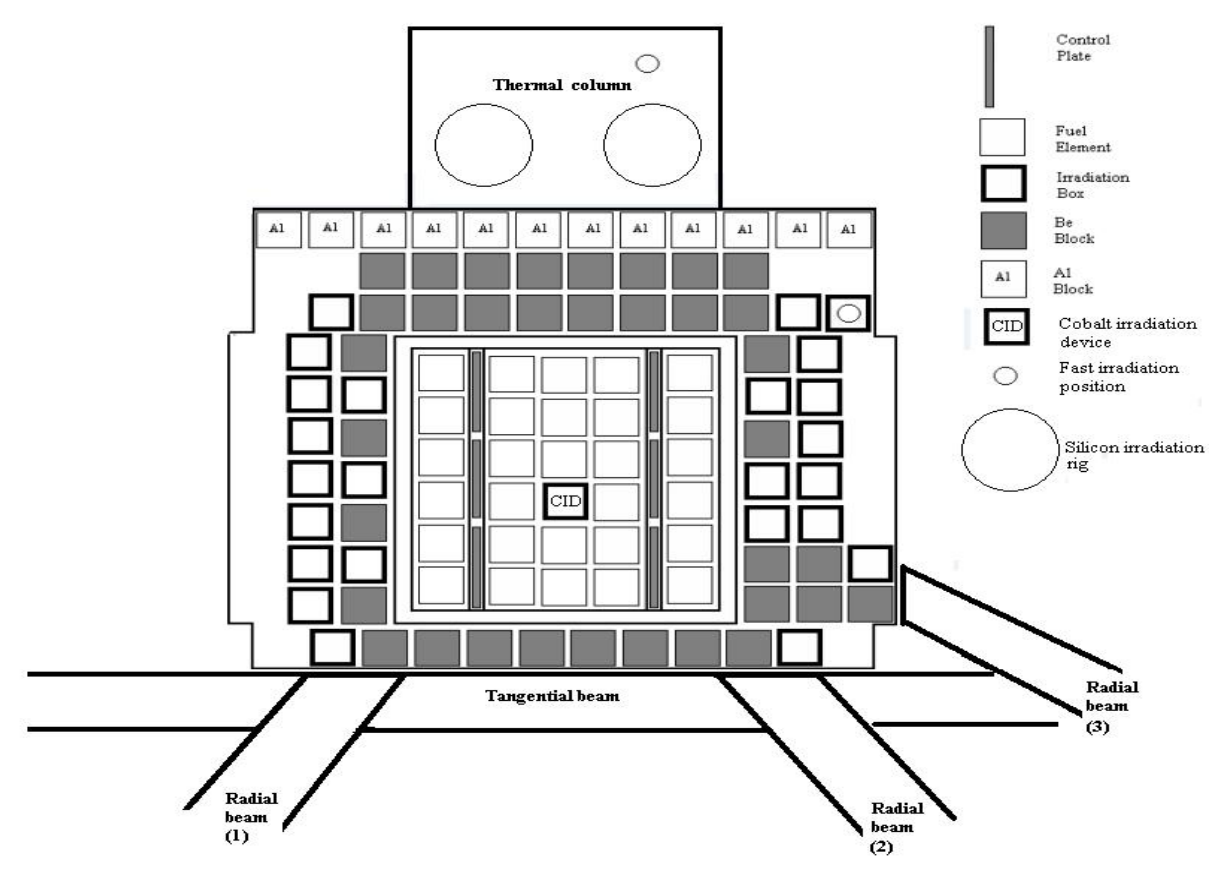

Figure 2: schematic diagram for the reactor core

\subsection{LOCA in the main open pool of the reactor}

LOCA is one of the anticipated accidents in nuclear reactor which results from rupture in the cooling system tubes and neutron beam tubes. The LOCA results in leakage of water from the pool and consequently decreasing the water level in the reactor pool accompanying with increasing the radiation dose level in the upper region of the pool tank and the reactor hall.

Normally, occurring LOCA would be accompanied with safe shutdown of the reactor (LOCWS) but taking the failure of the shutdown system (LOCNS) into account is important to predict the consequences of the worst case. Reducing the water level to the height close to the reactor core might result in increasing the water temperature to the boiling point and consequently causing of the core fuel damage. This point will not be discussed from the radiological view in this study since it needs another modeling attitude to be evaluated and it may be studied in a follow up research.

A flowchart was introduced, in figure 3 , to represent the sequence of the dose rate calculation at the top point of the main pool for the two LOCA cases. 


\subsubsection{LOCWS}

The water level in the main pool would be decreased in the main pool and the reactor core would be shut down safely due to the first or the second shutdown systems after the accident. Then, the power would be sharply dropped to the decay power mode that depends on the delayed neutrons and gamma rays from the fission products and the actinides. Then, the delayed neutrons and gamma rays would be evaluated using ORIGEN-S code to be introduced in the MAVRIC code to determine the dose rate as shown in the flowchart of figure 3.

\subsubsection{LOCNS}

In this case, the water level would be decreased in the main pool and the reactor core would be still on-operation due to failure in the shutdown systems. The prompt neutrons and gamma rays would be the main source that evaluated using KENO-VI code then introduced in the MAVRIC code to determine the dose rate as shown in figure 3.

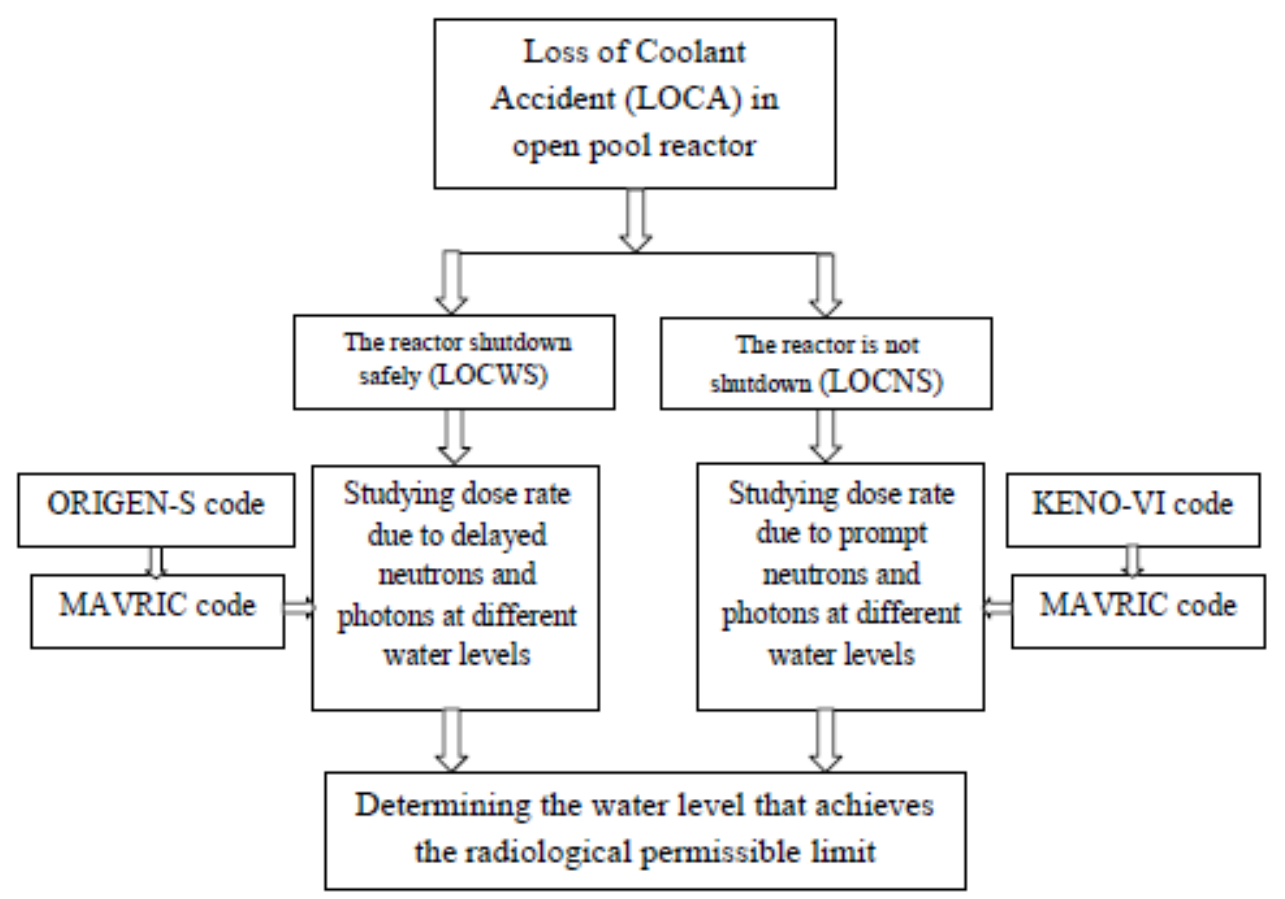

Figure 3: flowchart of studying the consequences of LOCA 
LOCA may be occurred after short or long time shutdown then the source intensity depends on the decay time from the core shutdown. The delayed neutrons and gamma rays would be evaluated at different decay times using ORIGEN-S code. In this study, the delayed neutrons and gamma rays would be evaluated at decay time ranging between 0 to $30 \mathrm{~d}$ from the reactor shutdown.

\subsection{Scale model}

Choosing the solution sequence in the Scale system depends on the type of LOCA accident. The solution models for LOCWS and LOCNS in SCALE 6.1 system are two-step approach using (ORIGEN-S and MAVRIC) and (KENO-VI and MAVRIC) respectively.

\subsubsection{Simulating the core source in LOCNS}

The first step is the estimation of the source distribution, done with the CSAS6 (Criticality Safety Analysis Sequence) control sequence, that uses the KENO-VI functional module as shown in figure 4. KENO-VI has been modified to accumulate the fission distribution over the non-skipped generations along calculating the core effective multiplication factor (k-eff). The fission distribution is collected on a three-dimensional Cartesian mesh, that overlays the core model, and is saved as a Monaco mesh source [4].

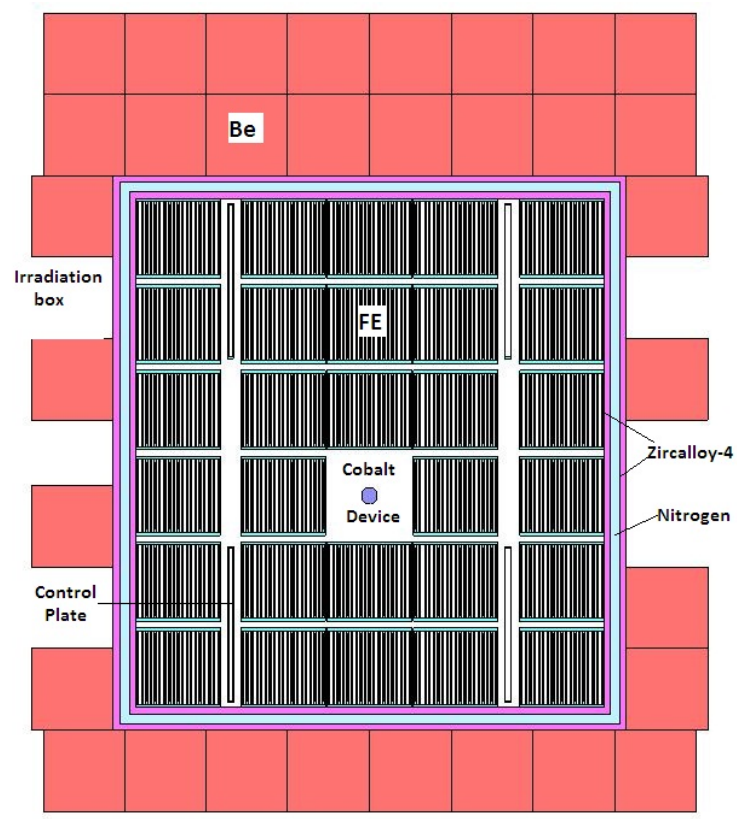

Figure 4: KENO-VI model for the reactor core. 
The mesh source is then used in the second step as a source term in MAVRIC. The total number of fissions is based on the total power released, $22 \mathrm{MW}$, during the normal reactor operation. The total fissions were set to $1.675 \mathrm{E}+18$ fissions with nu of 2.4396 . Fission photons were added to the source using the distribution for ${ }^{235} \mathrm{U}$. The total prompt neutrons and gamma rays intensities are $4.086 \mathrm{E}+18 \mathrm{n} / \mathrm{s}$, and $1.12 \mathrm{E}+18 \mathrm{p} / \mathrm{s}$ respectively during normal reactor operation at $22 \mathrm{MW}$ power.

Figure 5 is the meshview of the total sampled neutrons values obtained from KENO-VI model for all the fuel elements in the critical core. The sampled neutrons values would range between 5.05E-6 to 1.02E-5 depending on the fuel element position.
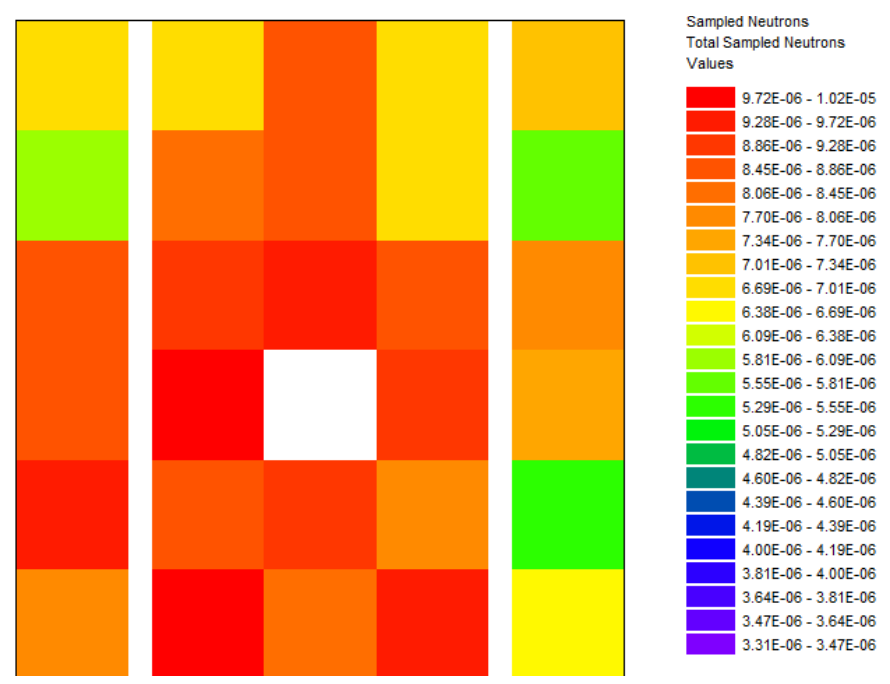

Figure 5: the total sampled neutrons values in the reactor core.

\subsubsection{Simulating the core source in LOCWS}

The first step is the estimation of the delayed neutrons and gamma rays in the reactor core using ORIGEN-S code. The reactor core was considered irradiating to power of $22 \mathrm{MW}$ and the neutrons and gamma rays intensity was introduced in $27 \mathrm{n} / 19 \mathrm{~g}$ energy spectrum at different decay times ranging from 2 min to $30 \mathrm{~d}$.

ORIGEN-S code was applied in the first step, for LOCWS, to get the delayed neutrons and gamma rays intensities in the reactor core at different decay times. Figure 6 shows the delayed photons and neutrons emitted from the reactor core after shutdown at decay times ranging from 20 $\min$ to $30 \mathrm{~d}$. The delayed neutrons intensity would be dropped from $2.45 \mathrm{E} 14$ to $1.27 \mathrm{E} 7 \mathrm{n} / \mathrm{s}$ at decay 
times of 2 and $20 \mathrm{~min}$. Then, it takes the value around 4.16E4 n/s for the decay times more than 20 min. The delayed gamma rays intensity would be dropped from $1.12 \mathrm{E} 18$ to $5.37 \mathrm{E} 16 \mathrm{p} / \mathrm{s}$ during decay time between 2 min and $30 \mathrm{~d}$.

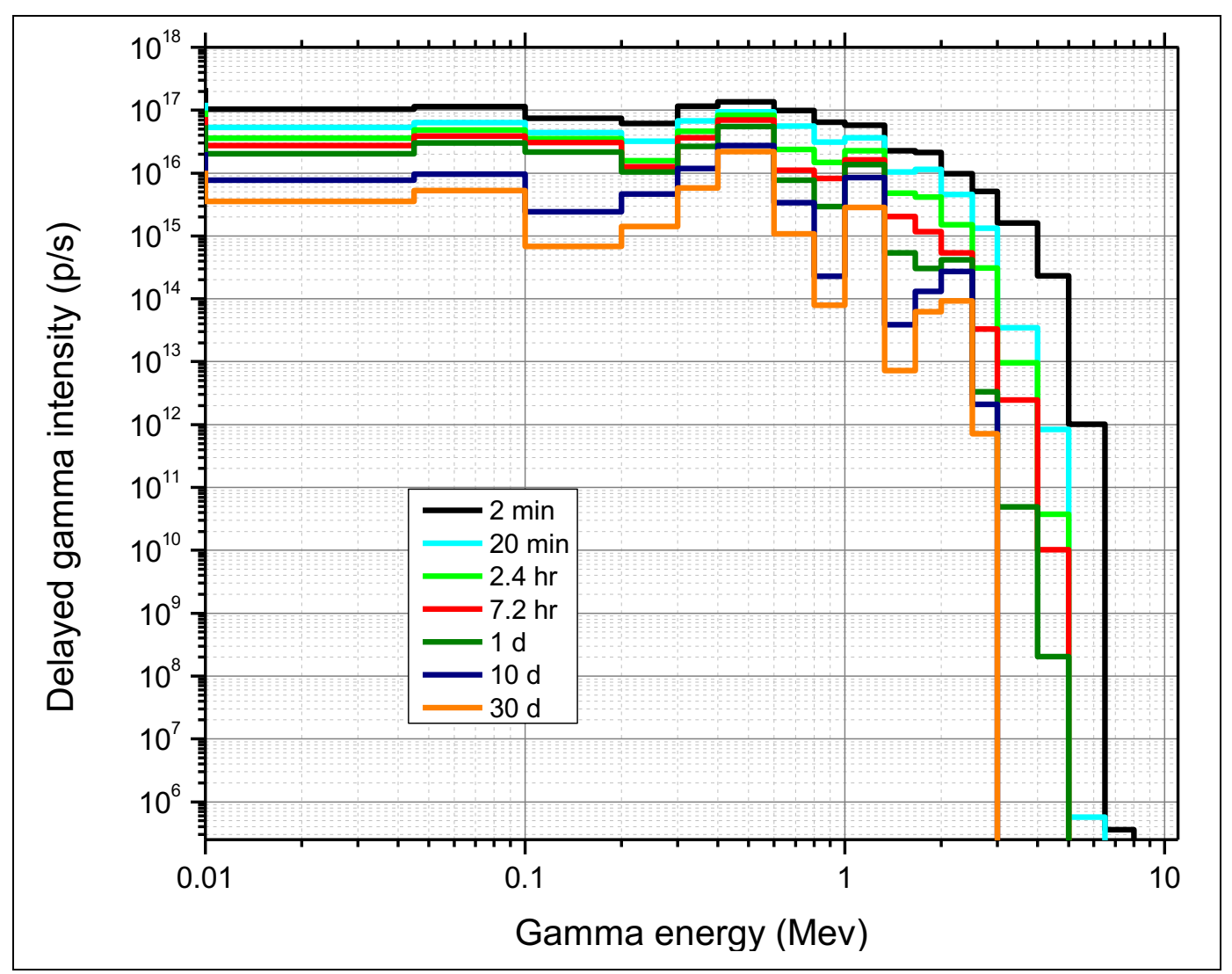




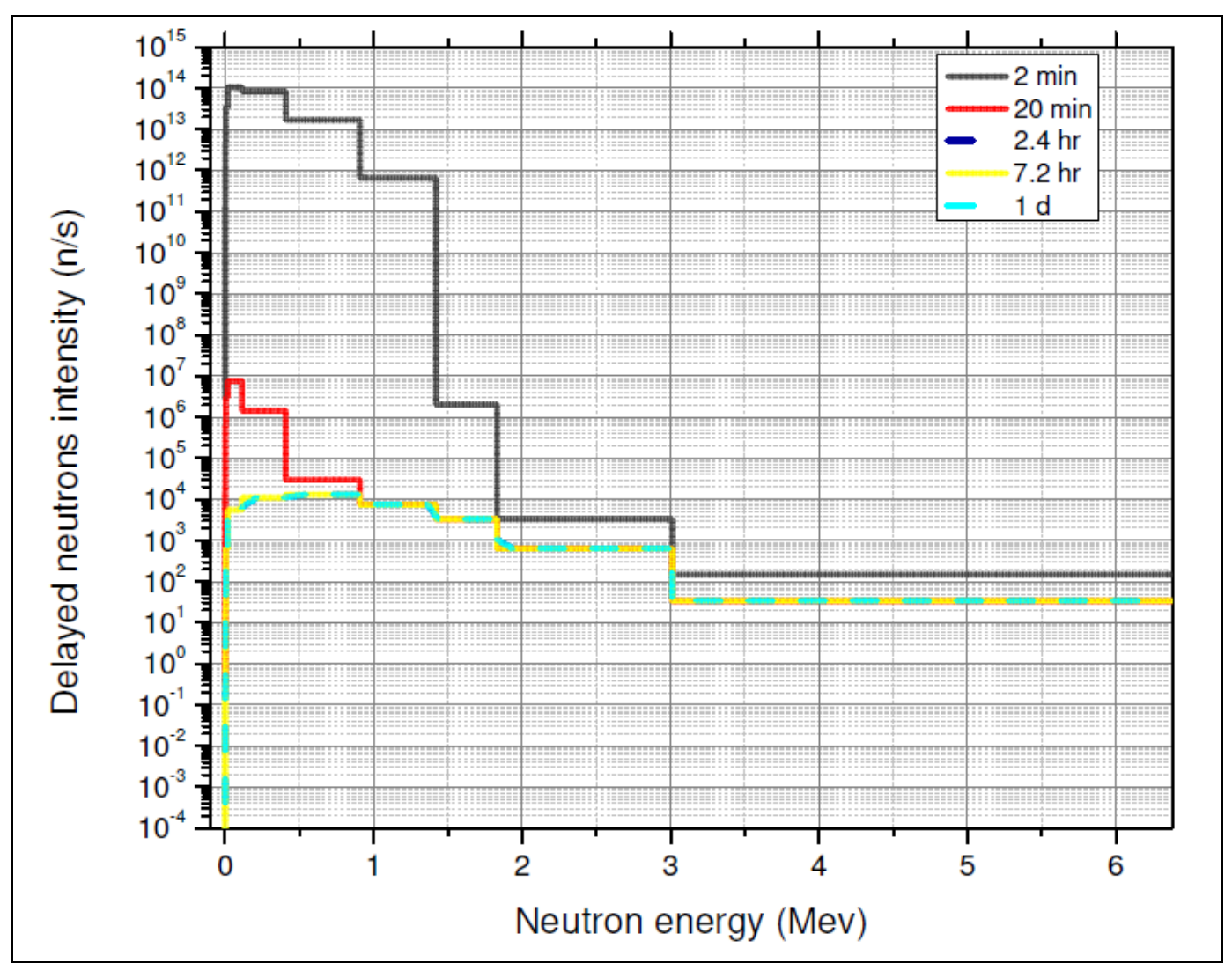

Figure 6: Delayed photon and neutrons intensity vs energy at different decay times

\subsubsection{Simulating the main pool during LOCA}

The reactor main pool that contains the reactor core under water surface was simulated using KENO-VI as shown in figure 7. In LOCNS case, the further neutron multiplication is prevented in the MAVRIC transport calculation. If further fissions were allowed, Monaco would add neutrons to its particle bank faster than they could be removed (since the system is near critical) and would never finish.

The MAVRIC calculations used the fission distribution mesh source that was produced by KENO-VI for LOCNS case, and delayed gamma rays and neutrons spectrums that was produced by ORIGEN-S code for LOCWS case. The ENDF/B-VII.0 27n/19g shielding library was used, in MAVRIC code [4]. The source for each case was placed in the same place of the reactor core.

For the importance maps in each MAVRIC calculation, a coarse mesh of about $15 \times 15 \times 30$ voxels was defined over the entire model including the core. The mesh planes included all of the significant material boundaries planes, and planes bounding (the reactor pool, the core, the shielding 
concrete, the detector, and horizontal plane at different levels to simulate the water level above the reactor core during LOCA).

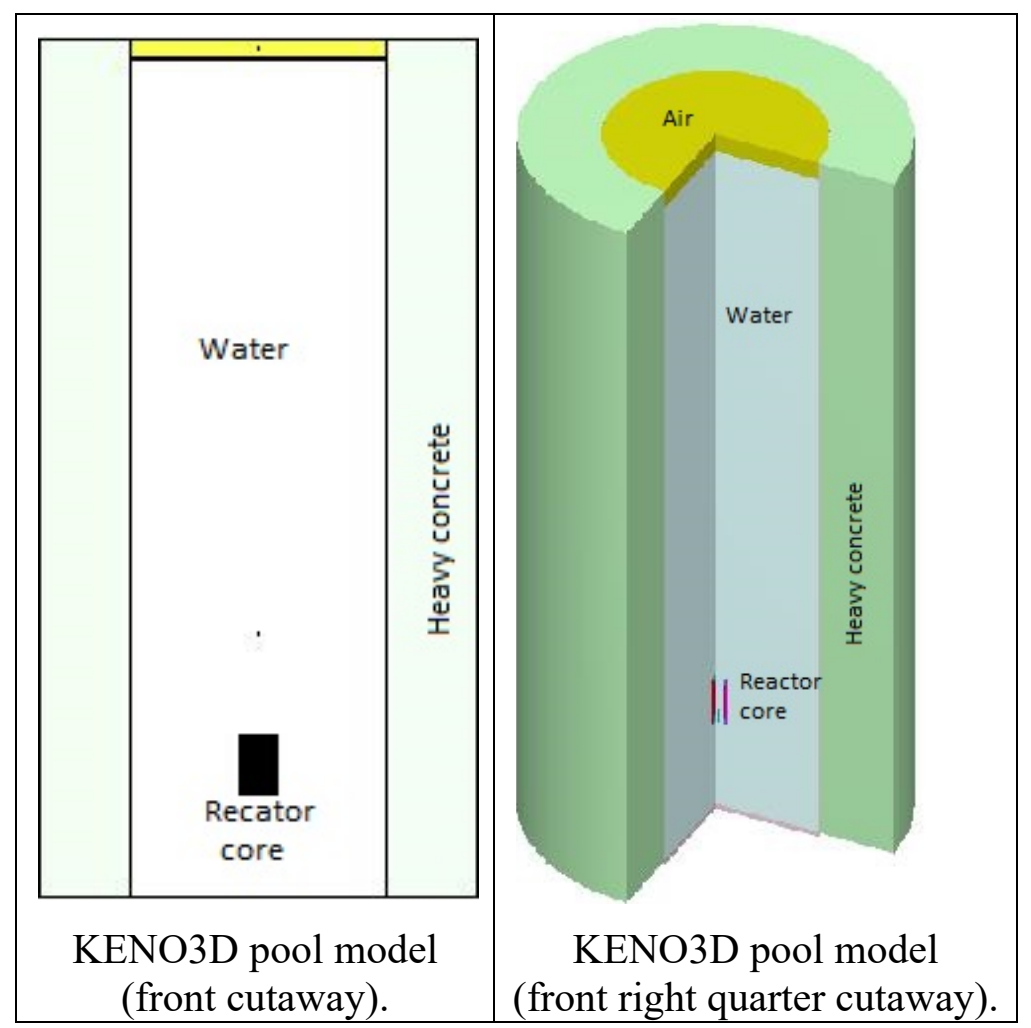

Figure 7: KENO-VI model for the reactor pool

\section{RESULTS AND DISCUSSION}

A point detector is located at the top of the main pool to determine the neutron and gamma ray dose rates using ICRU-57 conversion factors for neutrons and gamma rays [5]. Maximum of 50000 histories per batch with 10 numbers of batches were used at the maximum water level, normal condition in the reactor pool, for getting the dose rate calculation with the highest accuracy. The histories number would be decreased with decreasing the water level in the reactor pool owing to decreasing the water shielding thickness. Adjoint calculation was used also for determining the importance map for the reactor pool at each water level to increase the dose rate accuracy with decreasing the consumption time of calculations. 
The dose rate would be studied along the water level drop ranging from the normal level, $11 \mathrm{~m}$ above the core, to the water level at the top of the active core length for LOCNS as well as LOCWS at certain decay times as shown in figure 8. By decreasing the water level, the dose rate at the top point would be increased and the intersecting points between dose rate curves for all cases and horizontal line would represent the water levels that achieve the permissible dose rate limit (1.E-5 $\mathrm{Sv} / \mathrm{h})[6]$ at different LOCA cases.

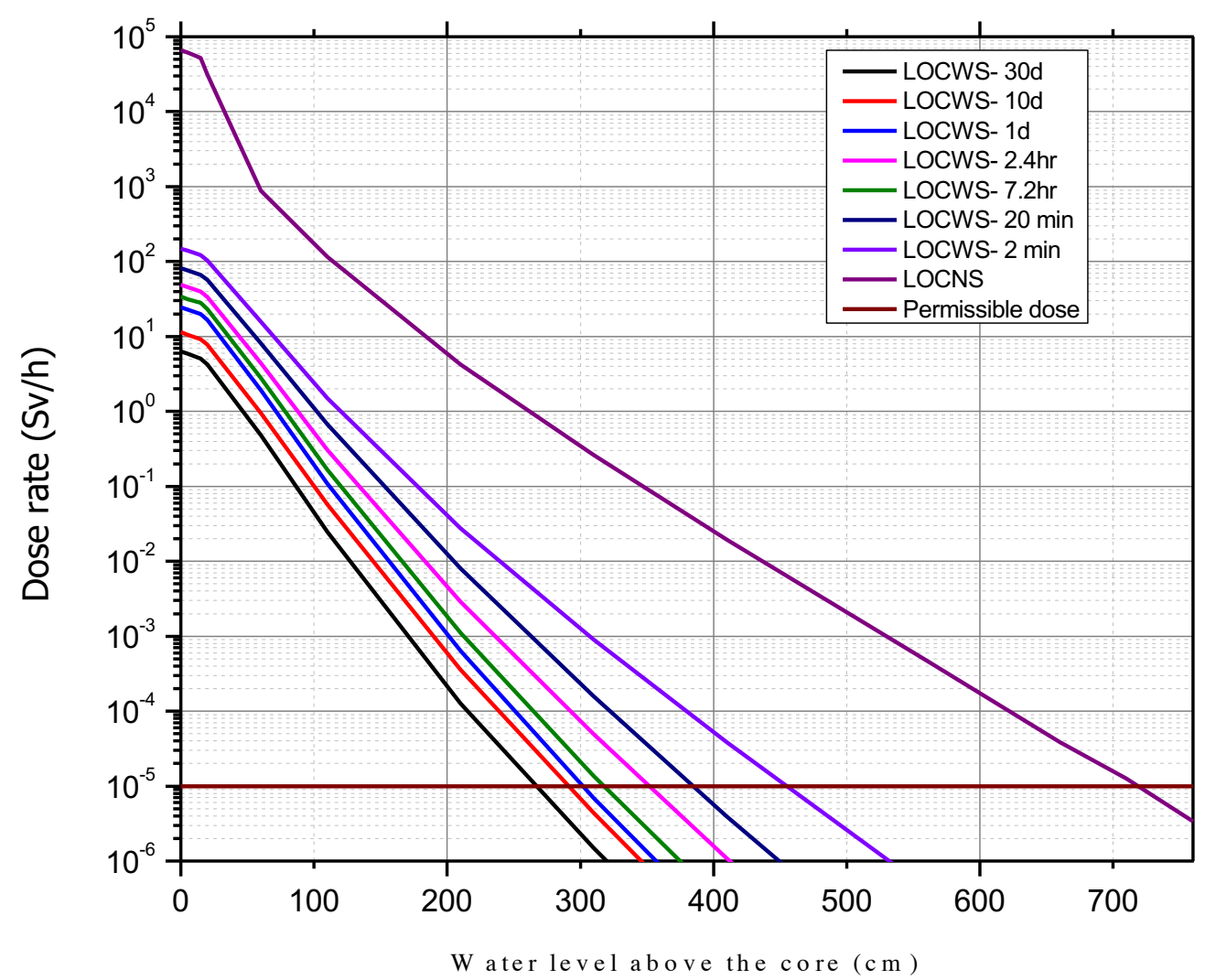

Figure 8: dose rate profiles vs water level during different LOCA types

\subsection{Case of LOCNS}

The neutron dose rate is the dominant in the region of water level less than $20 \mathrm{~cm}$ then it drops sharply to the value $1 . E-9$ at water level of $90 \mathrm{~cm}$ above the core. So, the total dose rate is mainly coming from the neutron contribution in water level less than $20 \mathrm{~cm}$. For water level more than 20 $\mathrm{cm}$ the total dose rate is coming from the prompt gamma ray and the contribution of prompt 
neutrons is negligible. The water level height that achieves the permissible limit would be $720 \mathrm{~cm}$ above the core in LOCNS case.

\subsection{Case of LOCWS}

The total dose rate for all LOCWS cases would be come from the delayed gamma rays. The maximum delayed neutrons dose rate is represented at the uncovered core for (LOCWS-2 min) case and it has a value of $2.4 \mathrm{~Sv} / \mathrm{h}$ which is much lower than the delayed gamma rays dose rate value $(147 \mathrm{~Sv} / \mathrm{h})$. Then, the dose rate for the cases between (LOCWS-20 $\mathrm{min}$ ) to (LOCWS-30 d) would be totally come from the delayed gamma rays.

The intersecting points between the dose rate curves of the cases from LOCWS-2 min to LOCWS-30 $\mathrm{d}$ and the horizontal line (represents the permissible dose rate limit) would be 455,385 , $350,320,300,290$, and $265 \mathrm{~cm}$ respectively, and these points represent the water levels that correspond the permissible limit at the top point. The water levels that achieve the permissible limit $(W L)$ would be represented versus the decay times $(D T)$ in figure 9.

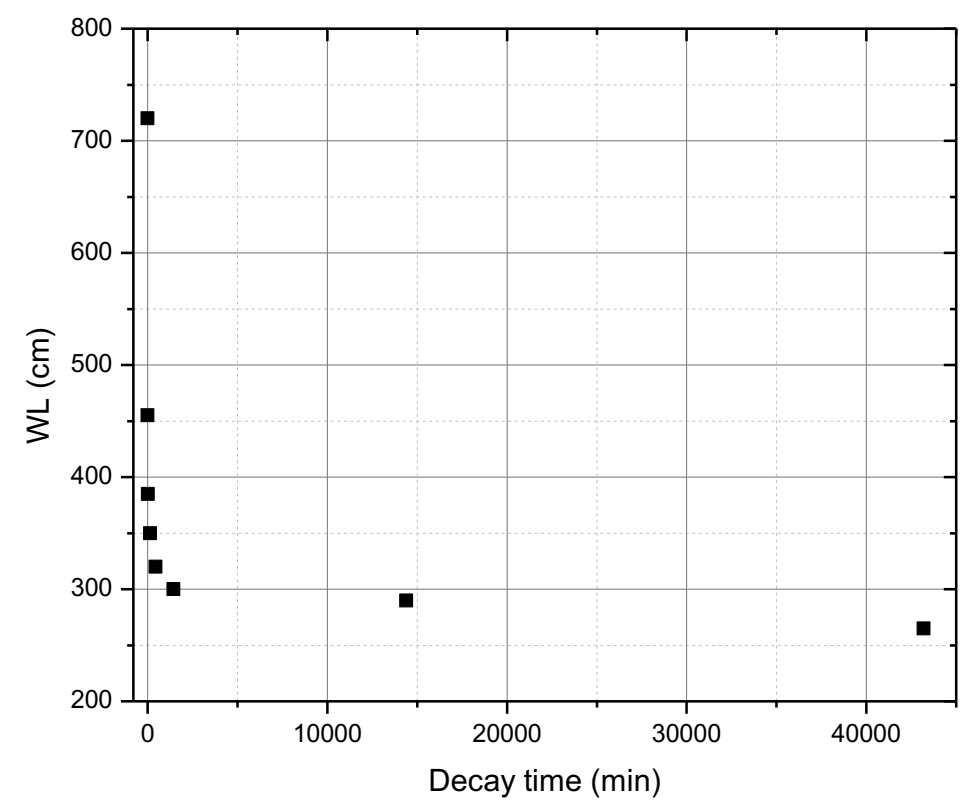

Figure 9: the $W L$ vs $D T$.

In order to introduce a relationship between the water level $(W L)$ that achieve the permissible limit versus the decay time $(D T)$ from the reactor shutdown, the data in figure 9 would be fitted by dividing it into two regions as shown in figure 10. 


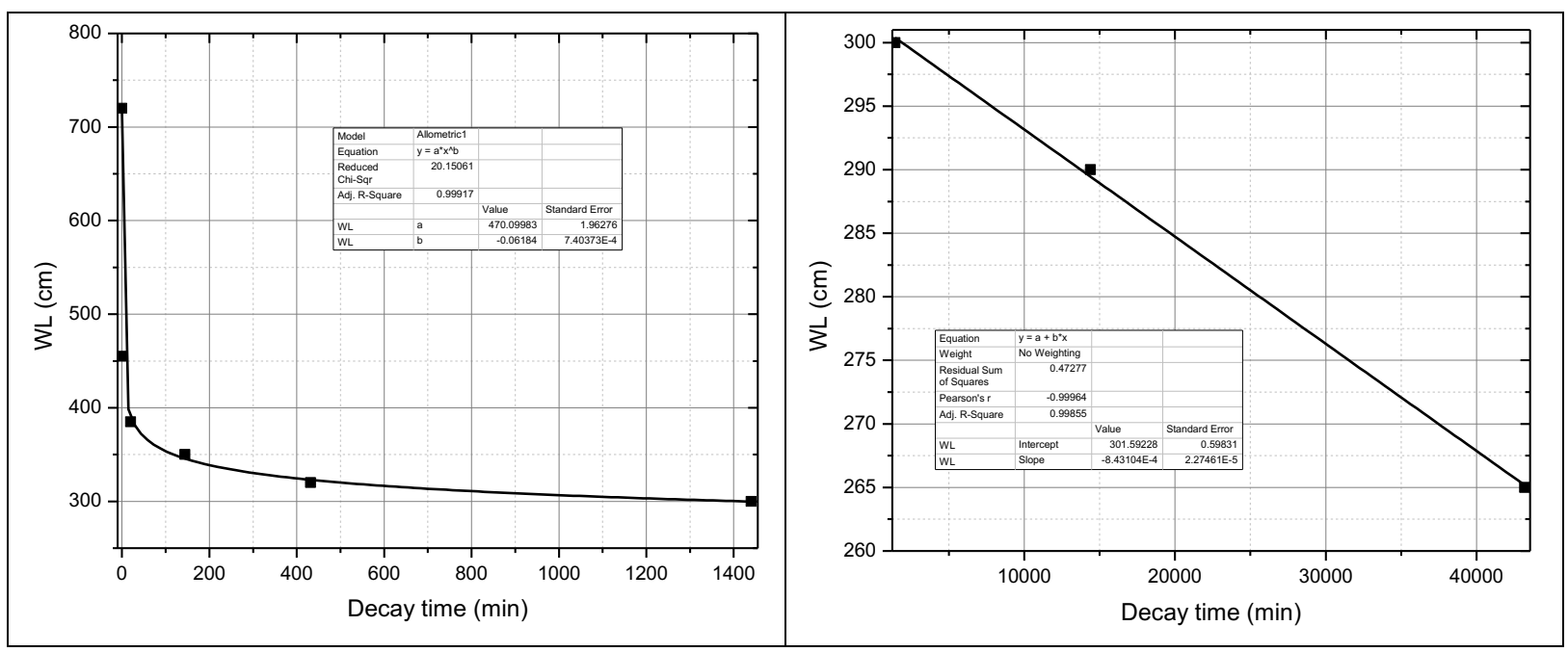

Figure 10: the fitted curves that represent the $W L$ vs $D T$

Each region has been fitted using Origin8.5/Origin-Lab software [7] and it was found that the following equations would represent the best fitting for the data plotted in figure 9:

$$
\begin{array}{cc}
W L(\mathrm{~cm})=470.1 \times(D T+0.001)^{-0.06184} & 0 \leq D T \leq 1440 \mathrm{~min} \\
W L(\mathrm{~cm})=301.59-8.43 \times 10^{-4} \times D T & 1440 \leq D T \leq 43200 \mathrm{~min}
\end{array}
$$

Since the water level that achieves the permissible limit was calculated at certain decay times, the fitted equations enable to expect the water level at any decay time in the range between 0 to $30 \mathrm{~d}$. For example, the water level that achieves the permissible limit at decay times of $0.5 \mathrm{~d}$ (720 min), and $5 \mathrm{~d}(7200 \mathrm{~min})$ would be $470.1 \times(720+0.001)^{-0.06184}=312.96 \mathrm{~cm}$, and 301.59-

$8.43 \times 10^{-4} \times(7200)=295.52 \mathrm{~cm}$ respectively.

The following table represents a comparison between the expected $W L$ using the fitted equations with those calculated using the MAVRIC model at different DT: 
Table 1: a comparison between the fitted with the calculated $W L$ at different $D T$.

\begin{tabular}{ccc}
\hline $\boldsymbol{D T}(\mathbf{m i n})$ & $\begin{array}{c}\boldsymbol{W L}(\mathbf{c m}) \text { using } \\
\text { Fitted equations }\end{array}$ & $\begin{array}{c}\boldsymbol{W L}(\mathbf{c m}) \text { using } \\
\text { MAVRIC model }\end{array}$ \\
\hline 0 & 720.47 & 720 \\
\hline 1 & 469.97 & --- \\
\hline 2 & 450.26 & 455 \\
\hline 5 & 425.47 & --- \\
\hline 10 & 407.62 & --- \\
\hline 20 & 390.52 & 385 \\
\hline 50 & 369 & --- \\
\hline 100 & 353.52 & --- \\
\hline 144 & 345.64 & 350 \\
\hline 432 & 322.94 & 320 \\
\hline 1440 & 300.37 & 300 \\
\hline 1600 & 300.24 & --- \\
\hline 2500 & 299.48 & --- \\
\hline 14400 & 289.45 & 290 \\
\hline 17000 & 287.26 & --- \\
\hline 42000 & 266.18 & --- \\
\hline 43200 & 265.17 & 265 \\
\hline
\end{tabular}

The comparison shows a good agreement between the fitted WL with those calculated using MAVRIC model so, the fitted equations are reliable to use in expecting the $W L$ during LOCA in open pool reactor of $22 \mathrm{MW}$ power that having the aforementioned characterizations.

\section{CONCLUSION}

Radiological dose rate resulting from LOCA was studied at the top point of the main pool of open pool-type reactor. The dose rate was determined for LOCA accompanied with safe shutdown as well as failure of shutdown systems. The dose rate was studied along water levels during LOCA. All calculations were conducted with ignoring the dose rate that results from the dissolved radioactive materials in the reactor pool water. Scale/ORIGEN-S and Scale/KENO-VI were used to determine the neutrons and gamma rays sources for safe shutdown and failure of shutdown systems respectively. Scale/MAVRIC code was used to determine the dose rate along the water levels and 
consequently to estimate levels that achieve the safety condition at the top point for different decay times after reactor shutdown. A fitted function was introduced to expect the water level that achieves the permissible limit as a function of the decay time ranging between 0 to $30 \mathrm{~d}$ after shutdown.

\section{REFERENCES}

[1] Abdelhady A., Radiological performance of hot water layer system in open pool type reactor, Alexandria Engineering Journal (2013).

[2] Scale: A Comprehensive Modeling and Simulation Suite for Nuclear Safety Analysis and De sign, ORNL/TM-2005/39 Version 6.1, June 2011.

[3] Abdelhady A. Dose rates from the accidental withdrawal of a fuel element from an open pool type reactor. J Nucl Eng Radiat Sci 2018; 4:021010-1.

[4] Thomas M. Miller, Douglas E. Peplow, GUIDE TO PERFORMING COMPUTATIONAL ANALYSIS OF CRITICALITY ACCIDENT ALARM SYSTEMS, ORNL/TM-2013/211.

[5] ICRU-57, "Conversion Coefficients for Use in Radiological Protection Against External Radia tion," International Commission on Radiation Units \& Measurements, Bethesda, MD (1998).

[6] ICRP (International Commission on Radiation Protection) (1991), Recommendations of the In ternational Commission on Radiation Protection, Pergamon Press, Oxford, England, ICRP Publication 60.

[7] Origin(Pro), Version 8.5 SR1. OriginLab Corporation, Northampton, MA, USA. 